\title{
La certification dans le domaine de la radioprotection
}

\author{
M. Fondeviole ${ }^{1,2, *}$, L. Jatteau ${ }^{2}$ et P. Vaucheret ${ }^{2}$ \\ ${ }^{1}$ GIIN, Groupe Intersyndical de 1'Industrie Nucléaire, Courbevoie, France. \\ 2 CEFRI, 39 rue Louis Blanc, CS 30080, 92038 La Défense cedex, France.
}

Reçu le 12 décembre 2017 / Accepté le 18 avril 2018

\begin{abstract}
Résumé - Cet article retrace l'historique du CEFRI, dès 1990 depuis sa création, jusqu'aux dernières évolutions réglementaires actuelles. Il confirme la démarche de recherche d'efficacité par une action continue de simplification administrative et de numérisation des processus, alliée à une grande compétence des intervenants. Est réaffirmée la volonté du CEFRI, créé par la profession, de conserver le niveau de qualité de la certification qui a fait ses preuves depuis maintenant plus de 20 ans; cette certification a participé sans aucun doute à aider les exploitants à garantir la sécurité des opérations en milieu hostile.

Abstract - Certification in the field of radiation protection. This article traces the history of CEFRI from its creation in 1990 till the last regulatory evolutions. Is confirmed the approach of research of efficacy by a continuous action of administrative simplification and digitalization of processes, allied with a large competence of intervening people. Is reaffirmed the willingness of CEFRI, created by the profession, to keep the level of quality of certification which has proven for more than 20 years. There is no doubt that this certification helped the operators guarantee the security of operations in hostile environment.
\end{abstract}

Keywords: exposed workers / radiological protection / audit / certification / third party / CEFRI / COFRAC / quality assurance / management systems / regulation

\section{Introduction}

Très vite, les industriels du nucléaire appelés à intervenir en zones hostiles ont compris la difficulté de ces travaux: l'organisation, les précautions et la compétence à acquérir pour réussir leur exécution parfaite le plus rapidement possible et ainsi protéger le personnel. Dans cette problématique, ils ont conçu la nécessité d'une formalisation d'un savoir-faire généralisé de tous les intervenants et sa reconnaissance.

\section{Origine et création du CEFRI}

Ainsi, les professions du nucléaire réunies au sein du GIIN, Groupe Intersyndical de l'Industrie Nucléaire, créèrent en 1990 le CEFRI, Comité français de Certification des Entreprises pour la Formation et le suivi du personnel travaillant sous Rayonnements Ionisants. Ont alors été associés comme membres fondateurs actifs les exploitants (CEA, COGEMA et EDF), la SFRP et comme membre consultant l'OPRI (Office de Protection contre les Rayonnements Ionisants, maintenant intégré au sein de l'ASN et de l'IRSN) : cela avait l'avantage de la cohérence globale, d'intérêts bien compris de toute la filière nucléaire. Dans un tel esprit, le statut ne pouvait être que celui d'une Association de type 1901, à but non lucratif: en effet, aucun soupçon d'intérêt commercial n'était ici envisageable vu les objectifs assignés, seule une indépendance financière sans aucune subvention étant recherchée comme garante d'indépendance. Très vite se sont associés le Ministère de la Défense, les médecins du travail et la CNAM (Caisse Nationale d'Assurance Maladie); enfin, il convient de noter que les Ministères du Travail et de l'Industrie ont également apporté leur accord et leur soutien à cette dynamique.

Il faut rappeler que le nucléaire avait subi alors deux profonds traumatismes avec les accidents de :

- Three Mile Island (28 mars 1979), les risques liés au nucléaire apparaissent aux populations bien qu'il n'y ait eu aucun relâchement significatif extérieur. Une conséquence a été la réflexion du passage de l'approche évènementielle à celle par état (APE $\left.{ }^{1}\right)$, plus systémique, lors d'incidents ou d'accidents d'exploitation;

- moins de 10 ans après, a lieu le très grave accident de Tchernobyl (26 avril 1986) qui a vraiment montré à la

\footnotetext{
* Auteur de correspondance : michel.fondeviole@cefri.fr.
}

\footnotetext{
${ }^{1}$ Approche par États.
} 
population les risques majeurs d'accidents possibles: une prise de conscience aigüe de la situation a alors lieu en France qui possède un très important parc de centrales nucléaires. Le nucléaire (entreprises, exploitants et administration) renforce ses règles pour plus de maîtrise des risques et de transparence : la pratique française devient très en avance en matière de radioprotection, en particulier sur les règles Euratom.

\section{Missions et objectifs}

Les objectifs assignés au CEFRI par les membres fondateurs sont ainsi très simples, clairs et très précis à la fois : nous les rappelons tels qu'ils apparaissent dans ses statuts dès sa fondation: "Le CEFRI a pour but de certifier les entreprises intervenantes, les organismes de formation et les entreprises de travail temporaire et toute organisation qui emploie du personnel en milieu ionisant. Les objectifs fondamentaux de la création du CEFRI sont au nombre de trois :

- contribuer pour le personnel à l'amélioration de la prévention des risques liés aux travaux dans les installations nucléaires ;

- permettre aux entreprises françaises intervenant sur les marchés du nucléaire de faire la démonstration objective des actions de prévention qu'elles mènent;

- améliorer pour les entreprises françaises intervenant sur les marchés du nucléaire leur accès aux marchés européens et internationaux $»$.

En pratique, il fallait donc s'assurer principalement de la formation et de l'information des travailleurs, de la bonne application par l'entreprise du Code du travail par la mise en place d'un système de management du risque dû à la radioprotection, enfin du suivi dosimétrique des travailleurs.

Ce système d'audit tierce partie, volontairement mis en place par la profession qui en surveille la qualité au niveau du Conseil d'administration du CEFRI, a parfaitement fonctionné de par la compétence de ses intervenants en ce domaine et a fait ses preuves, vu les résultats obtenus (niveau d'organisation des entreprises et mesures dosimétriques observées).

Ces objectifs tels que précisés à l'époque sont restés dans les statuts du CEFRI jusqu'à la parution de l'arrêté du 27 novembre 2013 : c'est-à-dire durant plus de vingt ans !

La démarche ALARA est définie et appliquée par EDF dès cette période: elle a, par exemple, été appliquée lors des remplacements des générateurs de vapeur de Dampierre 1 pendant la phase de préparation en 1988-1989 et de réalisation en 1990. Notons que ce principe est intégré dans la législation française dès 1988 .

Durant cette période, il faut noter une lente dégradation des résultats dosimétriques sur les sites français avec une année particulièrement mauvaise 1991 (voir Fig. 1 de doses collectives par réacteur).

Aussi, dès sa création en 1990, il a fallu rapidement mettre en ordre de marche le CEFRI tout nouvellement constitué. Les statuts structurant l'association sont édités, un règlement intérieur ainsi qu'un Manuel Qualité sont écrits assurant le cadre de l'activité objet de l'association; les commissions (technique et de certifications) prévues sont créées et une équipe permanente assure l'animation de l'ensemble. Elle est gardienne des règles éditées et appliquées dans l'association par l'ensemble des intervenants. Enfin, des auditeurs qualifiés par l'association sont identifiés et des équipes d'audit constituées.

Trois faits notables sont à souligner dans cette dynamique, toujours présente depuis près de 30 ans :

- le CEFRI a toujours, depuis ses débuts, fonctionné grâce au bénévolat de ses instances. Elles sont constituées de personnes reconnues par leur compétence dans le domaine de la radioprotection en particulier $\left(\mathrm{PCR}^{2}\right.$, responsables sûreté, organismes de formation...), désignées par les différentes entreprises participantes et cooptées par leurs pairs; ceci montre s'il était nécessaire, l'implication et l'intérêt de la profession dans la démarche initiée alors;

- soucieux de conserver l'indépendance financière du CEFRI, garante de l'impartialité des décisions de certification avec la qualité des intervenants, le CEFRI a très vite appliqué les règles comptables des associations et fait certifier ses comptes par des commissaires aux comptes ;

- enfin, de par la qualité de ses instances et leur structure, le CEFRI assure un service de qualité et libre de toute influence, en particulier celle de ses membres fondateurs.

\section{Période où la certification était volontaire}

Des dates particulières sont encore à signaler, sans prétendre à leur exhaustivité.

Le premier certificat du CEFRI date de 1992. Notons bien qu'il s'agissait alors d'un acte volontaire car aucune imposition n'existait à l'époque.

1993 : issu d'un travail conjoint entre les exploitants et les industriels, dont le GIIN, le système Carnax permet d'homogénéiser les procédures d'accès des intervenants extérieurs sur les sites nucléaires et de rassembler en un même lieu (la plateforme Carnax) et sur un même support pour l'intervenant (le carnet d'accès), l'ensemble des informations concernant ce dernier (état civil, employeur, qualification, habilitations, formations, service de santé au travail, etc.). À ce jour, Carnax enregistre 35000 personnes et 1750 entreprises.

1995: EDF est le premier exploitant à officialiser la démarche et à exiger une certification de l'entreprise et une qualification de son personnel. Les autres exploitants ont ensuite suivi plus ou moins rapidement.

Dans sa logique de positionnement et de reconnaissance, le CEFRI demande en 1998 à être audité par l'organisme extérieur, créé en 1994, à savoir le COFRAC ${ }^{4}$ qui devient une autorité administrative reconnue d'accréditation des organismes certificateurs.

Dans sa logique de positionnement et de reconnaissance, le CEFRI demande en 1998 à être audité par l'organisme extérieur créé en 1994 à savoir le COFRAC ${ }^{4}$ qui devient une autorité administrative reconnue d'accréditation des organismes certificateurs.

\footnotetext{
${ }^{2}$ Personne compétente en radioprotection.

${ }^{4}$ Comité français d'accréditation.

${ }^{4}$ Comité français d'accréditation.
} 


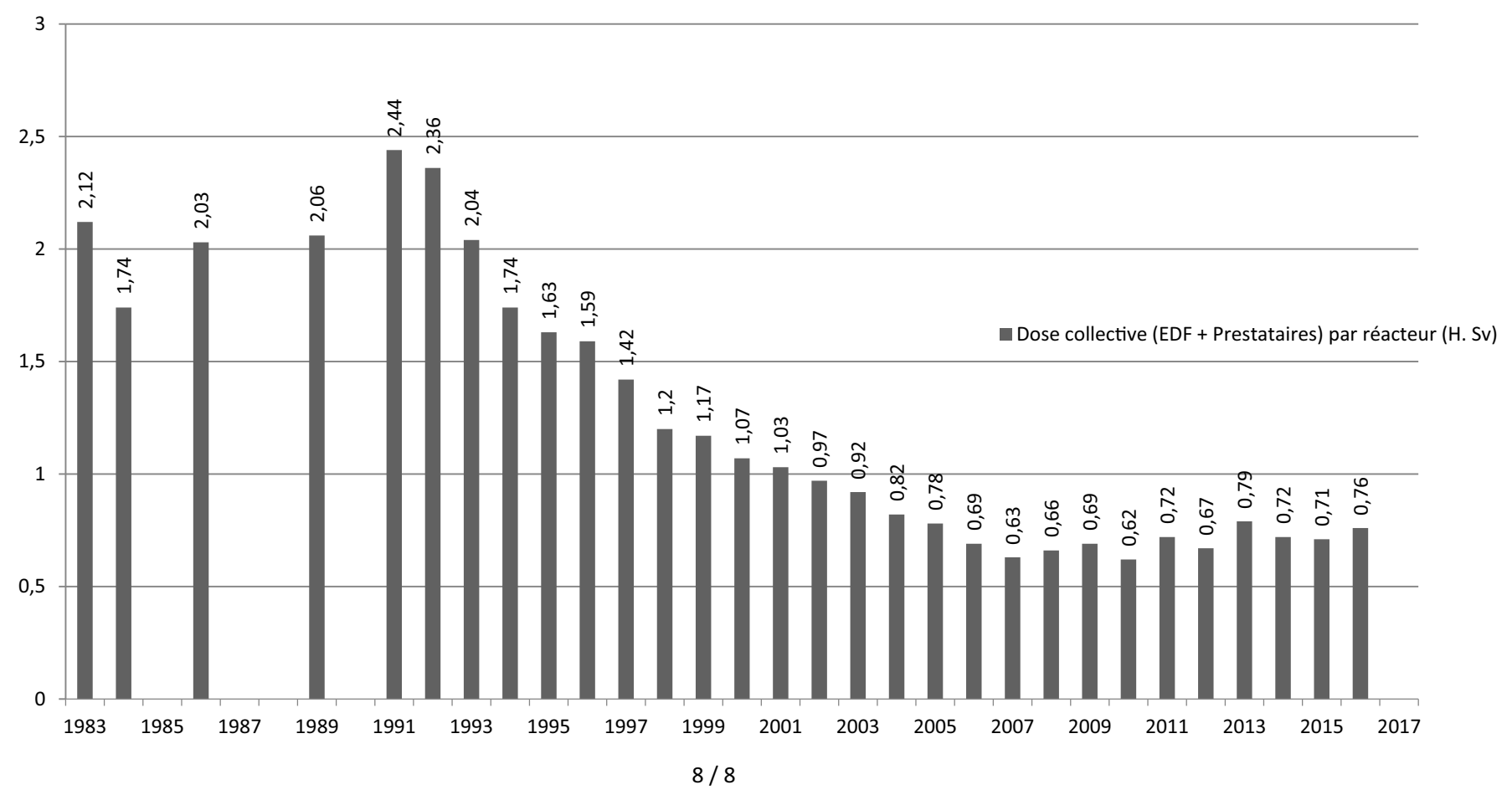

Fig. 1. Dose collective (EDF + Prestataires) par réacteur (H.Sv), de 1983 à 2016. Les doses collectives (EDF + Prestataires) par réacteur (H.Sv) sont représentées année par année, de 1983 à 2016, et pour les années où elles sont disponibles, à partir des sources suivantes : années 1983, 1986, 1989, 1992, 1993, 1994, 1995 : EDF faits marquants 2011 / Division production nucléaire (P.4); année 1984 : présentation de Gérard Cordier (EDF DPN), optimisation des activités et projet ALARA à EDF (P.1); année 1991 : présentation «Le management de la dosimétrie collective d'arrêt de tranche au niveau national à EDF » (auteurs : A. Caramel, J. Potoczek, F. Guerbeur [EDF - DSRE]) (P.2); années 1995 à 2015 : EDF Mémento de la radioprotection en exploitation / Chapitre 7 / Management de la radioprotection (P. 194).

Fig. 1. Collective dose (EDF and subcontractors) per reactor (H.Sv), from 1983 to 2016. The collective doses (EDF employees and subcontractors) per reactor (H.Sv) are presented per year from 1983 to 2016. Data were collected from the following sources: years 1983, 1986, 1989, 1992-1995: EDF/Nuclear production division. Highlights; 1984: Gérard Cordier (EDF/DPN) personal communication: "optimization activities and ALARA project at EDF"; 1991: A. Caramel, J. Potoczek, F. Guerbeur (EDF - DSRE) "collective dose management during reactor stop at the national level at EDF"; years 1995-2015: memorandum of radiation protection during operation/Chapter 7/Radiation protection management.

2005 : lancement de SISERI $^{5}$, qui remplace DOSIMO.

2006: création de l'Autorité de sûreté nucléaire (loi du 13/01/2006 relative à la transparence et à la sécurité en matière nucléaire). L'ASN assure au nom de l'État le contrôle de la sûreté nucléaire, de la radioprotection en France et l'information des citoyens pour « protéger les travailleurs, les patients, le public et l'environnement des risques liés aux activités nucléaires $»$.

Le CEFRI prend acte de cette règlementation en précisant son domaine d'activité : les INB.

Il est à noter à cette date qu'en dix ans, la dose moyenne reçue par les salariés des entreprises prestataires a été divisée par deux, avec une population d'intervenant concernés en hausse de $50 \%$ (Fig. 1). Ce beau résultat récompense les efforts de toute la profession sous la direction des exploitants, nous voulons croire que les processus de certification y ont aussi contribué sans aucun doute.

\footnotetext{
${ }^{5}$ Système informatique de surveillance de l'exposition aux rayonnements ionisants.
}

2011: accident de Fukushima (débuté le 11 mars 2011, séisme, puis raz-de-marée).

Tout au long de cette période, les référentiels du CEFRI ont évolué, dans le cadre des objectifs définis par les membres fondateurs, de façon à prendre en compte les retours d'expériences, aussi bien des exploitants que des certifiés. Des aspects essentiels audités portaient notamment sur la médecine du travail, la formation des intervenants en installations nucléaires et les plans de prévention.

Fin 2012, le CEFRI comptait 604 certificats actifs (dont 52 formateurs de PCR), avec une croissance progressive année par année depuis sa création.

\section{La certification devient réglementaire}

2013 marque une évolution majeure du contexte règlementaire avec la sortie de l'arrêté du 27 Novembre 2013 (entré en vigueur au $1^{\text {er }}$ Juillet 2015) relatif aux entreprises intervenant au sein d'établissements exerçant des activités nucléaires et aux entreprises de travail temporaire concernées 
par ces activités. Pour ce faire, la Direction Générale du Travail a travaillé sur la base de la pratique du CEFRI et consulté plusieurs organisations (CEFRI, COFRAC, membres du GPRADE ${ }^{6}$, partenaires sociaux...).

En ce qui concerne la certification et pour le CEFRI, certificateur historique, cela a pour conséquences principales :

- la certification qui était une décision de la profession devient une obligation légale ;

- la certification peut être prononcée par tout organisme accrédité COFRAC et donc indépendamment de la profession, une «concurrence» commerciale est ainsi instaurée;

- les bases de certification exigibles sont réduites à celles mentionnées dans l'Arrêté et uniquement. À noter que cette remarque ne porte évidemment aucun jugement de valeur sur les écarts avec la pratique CEFRI ancienne, certains ajouts comme les audits d'opération (audits sur site d'intervention) ayant été introduits par exemple pour la certification d'entreprises;

- enfin, s'il est maintenu la certification d'entreprises, l'Arrêté du 6 décembre 2013 entré en vigueur le $1^{\text {er }}$ juillet 2014 modifie les modalités de certification du Formateur de la personne compétente en radioprotection. La certification des organismes de formation de la PCR met fin à la certification de Formateur de PCR individuel. Des objectifs pédagogiques (au lieu d'un programme) sont donnés et des exigences très précises en ce qui concerne les personnes formées définies (niveaux, options, secteurs d'activité...);

- les entreprises de travail temporaires (ETT) continuent d'être concernées ;

- en 2013, l'arrêté SISERI oblige 1'employeur à communiquer toutes les informations nécessaires pour l'établissement de la carte individuelle de suivi médical et dématérialisation de celle-ci.

2014-2015, ces années ont été marquées par la refonte de tout le référentiel du CEFRI pour le rendre cohérent dans ses détails avec la nouvelle règlementation.

Il a fallu aussi gérer une phase de transition entre certificats prononcés avec l'ancienne documentation et la nouvelle, en accord avec les entreprises.
La nouvelle réglementation ayant conduit de nouvelles entreprises à devoir être certifiées (alors que cela ne leur était pas imposée précédemment), le CEFRI comptait, fin 2017, 662 clients certifiés (dont 18 organismes de formation de PCR).

Le CEFRI se tient aujourd'hui prêt pour les évolutions règlementaires à venir, notamment la loi de transition énergétique et la Transposition de la Directive EURATOM 2013-59 en cours dans la loi française, qui instaurera par exemple les $\mathrm{RPE}^{7} / \mathrm{RPO}^{8}$. Une série de fiches d'informations rédigées par Jean-Luc Godet et Thierry Lahaye paraissent actuellement dans la partie «La Vie règlementaire» du magazine Radioprotection et donnent un éclairage sur les principales nouvelles dispositions du Code du Travail devant entrer en vigueur le $1^{\mathrm{er}}$ janvier 2018.

\section{Conclusion}

Le CEFRI souhaite poursuivre la simplification administrative et la numérisation des processus. Les référentiels $\mathrm{E}$, I et F sont revus avec le support et le soutien des parties prenantes. En 2017, les QCM des organismes de formation sont mis à jour et un travail est en cours sur le référentiel de formation option $\mathrm{RN}$ (réacteur nucléaire). Un plus grand effort de communication est fait (nouveau site web, possibilité d'envoi des dossiers par email, participation à des salons). Le sens du client se développe avec la mise en place de questionnaires de satisfaction proposés aux organismes certifiés afin de toujours améliorer, vu du client, les processus administratifs qui entourent l'audit.

De nouveaux auditeurs, experts, évaluateurs pédagogiques et examinateurs sont intégrés chaque année afin de renforcer et renouveler les équipes d'audit.

La mesure de la dosimétrie des travailleurs du nucléaire démontre qu'elle est très bien maîtrisée. Ces résultats encourageants montrent, si cela était nécessaire, qu'il faut poursuivre dans la voie actuelle. Le CEFRI continuera à assurer son rôle dans cette dynamique générale.

\section{Contributeur}

Jean Marc Drewnowski.

Citation de l'article : Fondeviole M, Jatteau L, Vaucheret P. 2018. La certification dans le domaine de la radioprotection. Radioprotection 53(2): $97-100$

\footnotetext{
${ }^{6}$ Groupe permanent d'experts en radioprotection des travailleurs, du public et de l'environnement pour les applications industrielles et de recherche des rayonnements ionisants, ainsi que pour les rayonnements ionisants d'origine naturelle (Groupe permanent radioprotection et environnement).
}

\footnotetext{
${ }^{7}$ Radiation protection expert.

${ }^{8}$ Radiation protection officer.
} 